\title{
Volleyball best smashes angle based on numerical analysis method in the application of the game
}

\author{
Hongliang Yanga and Zhifen Jian \\ Institute of Physical Education, Huanggang Normal University, Huangzhou 438000, China
}

\begin{abstract}
For volleyball smash model, the analysis in the game in a different direction and Angle smashes volleyball after placement, get volleyball players hit range ball fall into the difficult to defense area. To establish the model, using differential equation can be solved by extremum method, Matlab simulation data, and attack the ball Angle range. Comprehensive comparison, it is concluded that the direction of the ball the best shot.
\end{abstract}

\section{Introduction}

Volleyball competition is a competition that fights for high and quick, viewed from the side, it is composed of field left and right two teams with six person each, a set is 25 points, the one that first gains the 25 points will win the set, two parties in competition process generally gain scores by spiking.

The paper researches on player instantaneous spiking status mainly from players spiking instantaneous leaping ability and instant hitting ball strength as well as orientations, defines how player hit ball so that can succeed in spiking good shot, so-called spiking good shot is the ball can succeed in dropping into opponent field after hitting. Due to field six players are arranged in symmetry, relatively middle defensive ability is stronger, which requires ball to be succeeded in dropping into opponent difficult defensive regions after player spiking, generally is the region in the back row.

\section{Volleyball hitting mechanical analysis}

Ideal model establishment: To simplify research process, regard volleyball as particle and during volleyball motion process, and don't consider volleyball rotation, air resistance [1] to volleyball motion influences.

Volleyball motion physical process: Regard volleyball motion after volleyball competition close spiking, far spiking and jump service as throwing. After athletes hitting, volleyball is thrown at horizontal speedv0, volleyball flight motions meet relative mechanics principles [2-4]. Volleyball flight motion time is up to volleyball height, volleyball flight motion horizontal distance is up to volleyball height and horizontal speedv0. Volleyball motion rules in different cases: Front row attacking extreme (maximum) speed studies. When ball is horizontally thrown from the height of $2.43 \mathrm{~m}$, drop point is just on the baseline. Similarly it can also solve ball's maximum speeds in different heights. So when make close spiking in the front row of men's volleyball, maximum speed is less than 12.78 $\mathrm{m} / \mathrm{s}$.

When jumping service minimum height is $h_{\text {min }}$, after service, volleyball drops in baseline.

Due to volleyball net two sides' sports horizontal displacements are the same, corresponding time is also the same. Therefore ratio between volleyball falling height in the right side of net and volleyball falling height in the left side of net is 1:3, jumping service minimum height is: $h_{\min }=\frac{4}{3} h_{n e t}=3.24 m$

Of course when spiking in volleyball competition, it can carry on cross spiking; when serving, make cross service to improve attacking speed and reduce opponent net interception opportunities. Such cases will not be further analyzed $[5,6]$.

\section{Establish models}

Establish coordinate axis in hitting point, during ball hit instant, assume sphere center is in the origin (as following Fig. 1 shows), then it gets speed components in $x, y, z$ axis $v_{x}, v_{y}, v_{z}$ :

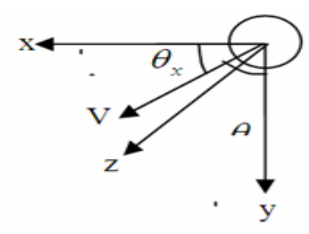

Figure 1. Hitting model.

\footnotetext{
${ }^{a}$ Corresponding author: ty-yhl@hgnu.edu.cn
} 
Success lies in the three components speeds sizes, and the three speeds components sizes is up to ball possessed speeds (that hitters' hitting strength and hitting orientation) after player hitting the ball, the problem requires to let ball to drop in difficulty defensive regions by defining $\theta_{x}, \theta_{y}$ angle range, so that problems solution can be summarized as objective functions when considering to succeed in dropping in difficult defensive regions each kinds of status: $\min \theta_{x}, \max \theta_{x}, \min \theta_{y}, \max \theta_{y}$.

$\left(L-l_{3}+l_{1}\right) \leq L_{x} \leq\left(L+l_{1}\right) ; L_{y} \geq H$; $-l_{b} \leq L_{z} \leq\left(L-l_{b}\right) . \quad x$ axis direction volleyball can succeed in dropping into regions of back row, ball values range in $x, y, z$ axis direction( use origin as 0 to define values sizes), as Fig.2.

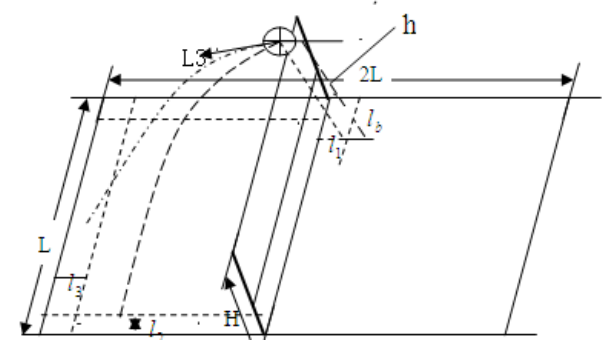

Figure 2. Spiking dropping region

Table 1. Spiking data

\begin{tabular}{|c|c|c|c|c|}
\hline Angle range & $\min \theta_{x}$ & $\max \theta_{x}$ & $\min \theta_{y}$ & $\max \theta_{y}$ \\
\hline $\begin{array}{c}\text { Back row difficult } \\
\text { defensive region }\end{array}$ & $0^{\circ}$ & $41.34^{\circ}$ & $71.82^{\circ}$ & $80.10^{\circ}$ \\
\hline $\begin{array}{c}\text { Longer boundary } \\
\text { difficult defensive } \\
\text { region }\end{array}$ & $41.40^{\circ}$ & $84.60^{\circ}$ & $73.80^{\circ}$ & $90.00^{\circ}$ \\
\hline $\begin{array}{c}\text { Shorter boundary } \\
\text { difficult defensive } \\
\text { region }\end{array}$ & $0^{\circ}$ & $84.60^{\circ}$ & $0^{\circ}$ & $90.00^{\circ}$ \\
\hline
\end{tabular}

By volleyball competition rules, we can know following data: volleyball court is generally in the length of $18 \mathrm{~m}$, width of $9 \mathrm{~m}$, two equal courts areas with the length of $9 \mathrm{~m}$, width of $9 \mathrm{~m}$ that are divided by center line; net height ( men's net height is $2.43 \mathrm{~m}$, women's net height is $2.24 \mathrm{~m} \mathrm{)}$; Ball specification is with circumference from $65 \mathrm{~cm}$ to $67 \mathrm{~cm}$. By international relative staff analyzing, during spiking, ball speed can arrive at $80 \mathrm{~km} / \mathrm{h}$, player spiking height arrives at above $3.5 \mathrm{~m}$; difficulty defensive region is back court area that nearly $1.2 \mathrm{~m}$, left and right are nearly $0.8 \mathrm{~m}$; we use player spiking in $1 \mathrm{~m}$ area far from center line, ball court gravity accelerated speed $g=9.8 \mathrm{~m} / \mathrm{s}^{2}$ to research on angle range that he can succeed:
We utilize women's volleyball spiking cases, use general ball speed $81 \mathrm{~km} / \mathrm{h}$, hitting point height of $3.5 \mathrm{~m}$ to estimate, by above solving, it gets conditions that $\theta_{x}, \theta_{y}$ should conform to, then it can get following three in equation sets, and then utilize computer to compute, use Matlab6.5, it can calculate and get following Table 1:

Then we can get optimal hitting angle is in the range of $\theta_{x}=\left[0^{\circ}, 41.34^{\circ}\right], \theta_{y}=\left[71.82^{\circ}, 80.10^{\circ}\right]$ that can spike good shot to difficult defensive regions.

\section{Conclusion}

By reasonable hypothesis and certain simplification, the paper applies qualitative analysis [7] and other methods to establish model, and then puts forward optimal hitting angle, which has certain actual significance in volleyball competition spiking direction, the model can be promoted and applied in differential equation establishment, solve its hitting angles, for service status, it can also solve its optimal hitting plan by establishing the model, but the model has some constraints, model considers ball falling as free fall motion, actually upper ball falling may not always like this, spiking is not surely required to spike into difficult defensive region so that can succeed in gaining scores, competitions should use flexible methods, aim at opponent difficult defensive points to spike, which can easier to get scores, so volleyball competition should use multiple tactics to win, model is just a simple hypothesis spiking method, in actual volleyball training, it has certain effects. From solving process, we can know hitting angle $\theta_{y}$ gets smaller, ball drop point will not prone to out of the bounds, so in volleyball spiking training, it can utilize the point to reduce spiking out-of-bounds rate, the establishment of the model is relative valuable in the aspect.

\section{References}

1. P. Terefenko and O. Terefenko, Journal of Coastal Research, 70, 706-711(2014).

2. C. F. Sun and X. Y. Yang, Journal of Xiaogan University, 23, 36-39(2003).

3. H. X. Feng, Y. C. Huang, Journal of Nanjing Institute of Physical Education: Natural Science, 6, 23-25(2007).

4. S. H. Cao, Journal of Beijing Union University, 5, 75-80(2007).

5. X. P. Ren, Journal of Zhejiang Normal University Natural Sciences, 35, 226-230(2012).

6. W. Jiang, Journal of Nanjing Institute of Physical Education: Natural Science, 10, 48-51(2011).

7. S. Sardar and Y. H. Lee, Advances in Industrial Engineering and Management, 4, 9-28(2015). 